\title{
DREAMM-2: Indirect Comparisons of Belantamab Mafodotin vs. Selinexor + Dexamethasone and Standard of Care Treatments in Relapsed/Refractory Multiple Myeloma
}

Thibaud Prawitz · Rakesh Popat · Attaya Suvannasankha - Grammati Sarri · Rachel Hughes · Feng Wang · Cosmina Hogea $\cdot$ Shannon Allen Ferrante $\cdot$ Boris Gorsh $\cdot$ Jenny Willson · Venediktos Kapetanakis

Received: April 20, 2021 / Accepted: August 3, 2021 / Published online: September 24, 2021

(C) The Author(s) 2021

\section{ABSTRACT}

Introduction: Single-agent belantamab mafodotin (belamaf; BLENREP) demonstrated deep and durable responses in patients with relapsed/ refractory multiple myeloma and $\geq 3$ prior lines of therapy, including an immunomodulatory

Supplementary Information The online version contains supplementary material available at https:// doi.org/10.1007/s12325-021-01884-7.

T. Prawitz

Evidence Synthesis, Modeling, and Communication Group, Evidera, Paris, France

R. Popat

NIHR/UCLH Clinical Research Facility, University

College London Hospitals, NHS Foundation Trust, London, UK

\section{A. Suvannasankha}

Indiana University Simon Cancer Center and

Roudebush VAMC, Indianapolis, IN, USA

G. Sarri · V. Kapetanakis $(\bowtie)$

Evidence Synthesis, Modeling, and Communication Group, Evidera, The Ark, 201 Talgarth Rd, Hammersmith, London W6 8BJ, UK

e-mail: Venediktos.Kapetanakis@evidera.com

R. Hughes

Evidence Synthesis, Modeling, and Communication

Group, Evidera, San Francisco, CA, USA agent, proteasome inhibitor, and anti-CD38 antibody (DREAMM-2; NCT03525678).

Methods: At the time of this study, STORM Part 2, NCT02336815 (selinexor plus low-dose dexamethasone; sel + dex) was systematically identified as the only feasible comparator to the DREAMM-2 cohort. Matching-adjusted indirect comparisons (MAIC) evaluated efficacy and safety of belamaf $(2.5 \mathrm{mg} / \mathrm{kg} ; n=97)$ versus sel $+\operatorname{dex} \quad(80 \mathrm{mg}+20 \mathrm{mg}, \quad$ respectively; $n=123)$. Populations were weighted for clinically validated effect modifiers and prognostic factors. Outcomes included overall survival

F. Wang · C. Hogea · S. A. Ferrante - B. Gorsh

Value Evidence and Outcomes, GlaxoSmithKline,

Upper Providence, PA, USA

J. Willson

Value Evidence and Outcomes, GlaxoSmithKline,

London, UK

Present Address:

G. Sarri

RWE Strategy Lead, Visible Analytics, London, UK

Present Address:

C. Hogea

Bristol Myers Squibb, Greater Philadelphia, PA, USA 
(OS), progression-free survival (PFS), duration of response (DoR), overall response rate (ORR), time to response (TTR), and safety. The relative efficacy of belamaf versus standard of care (SoC) on OS was estimated by a Bucher indirect treatment comparison using the MAIC-adjusted hazard ratios (HR) for OS of belamaf (DREAMM-2) versus sel + dex (STORM Part 2) and a HR adjusted for refractoriness to carfilzomib and high-risk cytogenetics of sel $+\operatorname{dex}$ (STORM) versus SoC (MAMMOTH).

Results: Belamaf demonstrated improved OS (HR 0.53; 95\% confidence interval 0.34, 0.83; $p=0.005)$ and DoR $(0.41 ; 0.21,0.83 ; p=0.013)$ versus sel + dex. There were no statistically significant differences in ORR, TTR, and PFS. Belamaf had a favorable safety profile for most evaluable hematologic (any-grade, Grade 3-4) and non-hematologic (any-grade) adverse events versus sel + dex. Significantly improved OS was observed with belamaf versus SoC $(0.29$; $0.16,0.54 ; p<0.001)$.

Conclusion: Single-agent belamaf represents a new treatment option for triple-class refractory patients with RRMM.

Keywords: Belamaf; Indirect treatment comparison; MAIC; MAMMOTH; Matchingadjusted; RRMM; Selinexor; Survival

\section{Key Summary Points}

There is a high unmet need for treatment for triple-class refractory patients with relapsed refractory multiple myeloma (RRMM); belantamab mafodotin was recently approved for clinical use in this patient population

Comparative effectiveness and safety of belantamab mafodotin versus relevant therapies and standard of care (SoC) in RRMM have not yet been established
In the absence of head-to-head comparisons, data from separate studies with similar designs, definitions, and patient populations can be evaluated via indirect treatment comparisons such as matching-adjusted indirect comparison

At the time of the study, the indirect treatment comparison revealed selinexor + dexamethasone in the STORM Part 2 study as the only feasible comparator to belantamab mafodotin in the DREAMM-2 study

The analyses demonstrated improved overall survival (OS) and duration of response as well as favorable safety profile for most evaluable adverse events with belantamab mafodotin versus selinexor + dexamethasone and a significantly improved OS with belantamab mafodotin versus SoC

\section{INTRODUCTION}

Multiple myeloma (MM) accounts for approximately $1 \%$ of all cancers and $15 \%$ of hematologic malignancies with an annual incidence of 86,000 new cases globally $[1,2]$. Despite major advances in treatment, MM remains an incurable disease, which requires multiple lines of therapy due to relapse [2, 3]. Available treatments for relapsed refractory MM (RRMM) include immunomodulatory drugs (e.g., lenalidomide and pomalidomide), proteasome inhibitors (PIs; e.g., carfilzomib and ixazomib), monoclonal antibodies (mAbs) targeting CD38 (e.g., daratumumab), and signaling lymphocytic activation molecule F7 (SLAM7; e.g., elotuzumab), the nuclear export protein inhibitors (XPO1; selinexor), alkylators, and steroids [2-4]. After initial response, patients eventually relapse, and each subsequent relapse is associated with cumulative treatment toxicity and a shorter duration of response, as patients develop refractory disease due to multiple drug resistance mechanisms [2, 5-7]. Patients with RRMM that is refractory to immunomodulatory 
agents, PIs, and an anti-CD38 antibody have a particularly poor prognosis $[2,8]$. Therefore, novel therapies with alternative modes of action are needed for this population with a high unmet need.

Belantamab mafodotin (belamaf; BLENREP; GSK2857916) is a first-in-class antibody-drug conjugate (ADC) that targets B-cell maturation antigen (BCMA) $[9,10]$. It comprises a humanized afucosylated anti-BCMA mAb conjugated to a cytotoxic payload monomethyl auristatin $\mathrm{F}$ (MMAF) by a protease-resistant mc linker [9]. Belamaf binds to BCMA and eliminates MM cells by a multimodal mechanism of action, including delivery of MMAF to MM cells, immune-independent ADC mediated apoptosis, and release of markers characteristic of immunogenic cell death as well as immunedependent mechanisms of action such as antibody-directed cellular cytotoxicity/phagocytosis $[9,10]$.

In the Phase II, single-arm DRiving Excellence in Approaches to Multiple Myeloma 2 (DREAMM-2 study; NCT03525678), multiply relapsed patients who received single-agent belamaf $2.5 \mathrm{mg} / \mathrm{kg}$ every 3 weeks showed an overall response rate (ORR) of $32 \%$, estimated median duration of response (DoR) of 11 months, overall survival (OS) of 13.7 months, and median progression-free survival (PFS) of 2.8 months at a median follow-up of 13 months $[9,11]$. On the basis of the DREAMM-2 study, single-agent belamaf $(2.5 \mathrm{mg} / \mathrm{kg})$ was recently approved in the USA and European Union for the treatment of adult patients with RRMM who have received at least four prior therapies including an anti-CD38 $\mathrm{mAb}$, a PI, and an immunomodulatory agent $[12,13]$

Demonstration of added value is important for novel treatments through comparative evaluations of efficacy and safety, which will inform on cost-effectiveness and enable decisions on payer interactions, clinical care, and reimbursement coverage. In the absence of head-to-head comparisons, data from separate studies can be evaluated via indirect treatment comparisons (ITC). ITC through network metaanalysis is not feasible for single-arm studies because of the network of evidence being disconnected [14]. Instead, population-adjusted ITCs are applicable in this setting, as recommended by the National Institute for Health and Care Excellence (NICE) Decisions Support Unit (DSU) [14].

Matching-adjusted indirect comparisons (MAIC) are a form of population-adjusted ITC that can be used to compare trials with similar designs, definitions, and patient populations. The MAIC method relies on weights assigned to patients in the trial, for which individual patient-level data are available, to match aggregate baseline data from comparator trials, thereby removing population differences that could bias comparisons of treatment outcomes. This provides important information to contextualize data from single-arm studies.

The goal of this post-hoc analysis of the DREAMM-2 study was to conduct ITC of belamaf versus relevant comparators and standard of care (SoC) in similar patient populations. A systematic literature review (SLR) was conducted to identify relevant comparator studies and is being submitted for publication [15]. Results of the SLR were used to assess the feasibility of conducting an ITC using the MAIC method to compare the efficacy and safety of belamaf versus selinexor data from Selinexor Treatment of Refractory Myeloma (STORM) Part 2 [16]. A Bucher ITC analysis was then conducted using the MAIC results for the OS of SoC in a subset of patients in the Monoclonal Antibodies in Multiple Myeloma: Outcomes after Therapy Failure (MAMMOTH) study who were refractory to a PI, an immunomodulatory agent, and daratumumab $[8,17,18]$.

\section{METHODS}

\section{Inclusion Criteria, Study Selection, and Data Sources}

This article is based on previously conducted studies and does not contain any new studies with human participants or animals performed by any of the authors. The DREAMM-2 study enrolled patients with RRMM treated with $\geq 3$ prior lines of therapy, who were refractory to an immunomodulatory agent and PI, and 
refractory and/or intolerant to an anti-CD38 $\mathrm{mAb}$ [9]. In this analysis, data from the 13-month follow-up of the DREAMM-2 study were used (cutoff date: January 31, 2020) [9]. An SLR was conducted in Embase, Medline, and MEDLINE In-Process, Cochrane Collection Central Register of Clinical Trials (CENTRAL), the Database of Abstracts of Reviews of Effects (DARE), Cochrane Database of Systematic Reviews (CDSR), and National Institute for Health Research-health technology assessment (NIHR-HTA) to identify suitable evidence for comparator treatments. Studies, including reports from previously published SLR, randomized clinical trials, single-arm studies, or observational studies conducted on prospective or retrospective evidence, published between January 2008 and March 2019, that enrolled patients with $\mathrm{RRMM}$ who received $\geq 3$ prior lines of therapy were included in the review (Supplementary Methods).

Of the studies identified by the SLR, only the STORM (NCT02336815) Part 2 study [16] was comparable with the DREAMM-2 study in study design, baseline patient and disease characteristics, including prior anti-CD38 therapy exposure, and definitions of outcomes (as per the International Myeloma Working Group [IMWG] 2016 efficacy criteria [19]).

STORM was a multicenter, open-label, Phase II study of selinexor (sel; XPOVIO ${ }^{[\mathrm{R}]}$ ) $80 \mathrm{mg}$ (orally [PO]) plus dexamethasone (dex) $20 \mathrm{mg}$ PO, both administered twice weekly for 4-week cycles $[16,20]$. The study was conducted in patients with RRMM that were refractory to at least one PI, one immunomodulatory agent, and daratumumab, glucocorticoids, and last treatment. Although in DREAMM-2, the inclusion criteria specified refractory and/or intolerant status to an anti-CD38 $\mathrm{mAb}$, all patients who enrolled in the $2.5 \mathrm{mg} / \mathrm{kg}$ group were refractory to an anti-CD38 $\mathrm{mAb}$ at baseline. Patient-level data from the DREAMM-2 study $[9,11]$ (data on file, 13-month follow-up manuscript in preparation) and multiple sources of efficacy and safety data for STORM Part 2 were used in this evaluation (Table 1) $[9,16,20-23]$.

At the time of the SLR, no additional studies were found that were comparable with the
DREAMM-2 population. However, a search for updated results on the STORM Part 2 study conducted after the SLR identified a publication comparing a cohort of the STORM Part 2 study versus a real-world cohort from the MAMMOTH study [17], which could facilitate an ITC of belamaf versus SoC. The MAMMOTH study was a retrospective study of patients with disease refractory to anti-CD38 [8]. The inclusion criteria of the subcohort of the MAMMOTH study reported in Costa et al. (2019) were similar to those of STORM Part 2 (Table 1), facilitating comparison between sel + dex and SoC [17]. Given the available published information, a Bucher ITC using the MAIC-adjusted hazard ratios (HR) for OS of belamaf versus sel + dex, and an HR adjusted for refractoriness to carfilzomib and high-risk cytogenetics of sel + dex versus SoC, was used to determine the relative efficacy of belamaf versus SoC from the MAMMOTH study on OS [17]. This article is based on previously conducted studies and does not contain any studies with human participants or animals performed by any of the authors.

\section{MAIC and Bucher ITC Methodology}

Due to the absence of a connected network for these single-arm randomized studies, unanchored MAIC of belamaf versus sel + dex was performed by following guidelines from the NICE DSU for population-adjusted ITC [14]. Propensity score-like regression was used to calculate for each patient in DREAMM-2 a predicted probability of inclusion in the STORM Part 2 study based on patient characteristics (Table 2). These estimated probabilities were then used as statistical weights and applied to the DREAMM-2 population to balance DREAMM-2 and STORM Part 2 on the factors included in the regression model (by weighting the DREAMM-2 population). These weights were also used to calculate the effective sample size (ESS) corresponding to population size of the weighted cohort of patients who received belamaf.

Adjustments were made for imbalances of known treatment-effect modifiers identified 


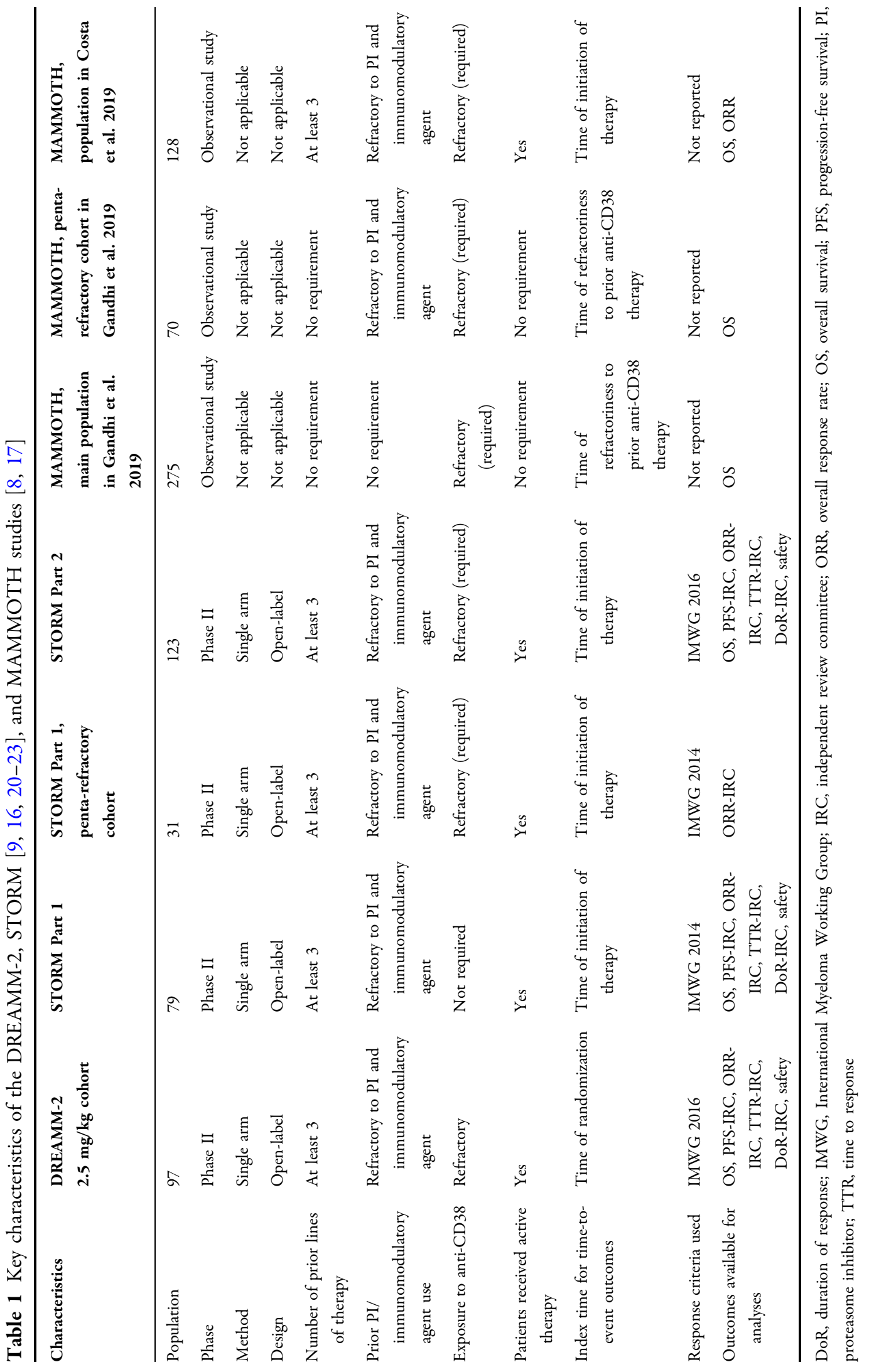


Table 2 Prognostic factors and treatment-effect modifiers included in the MAIC analysis

\begin{tabular}{|c|c|c|c|c|c|}
\hline Factors & $\begin{array}{l}\text { Prognostic factor } \\
\text { according to } \\
\text { clinical experts }\end{array}$ & $\begin{array}{l}\text { Effect-modifier } \\
\text { according to } \\
\text { clinical experts }\end{array}$ & $\begin{array}{l}\text { Prognostic factors } \\
\text { in the DREAMM- } \\
2 \text { data }\end{array}$ & $\begin{array}{l}\text { Available for } \\
\text { comparison in } \\
\text { STORM Part } 2\end{array}$ & $\begin{array}{l}\text { Included in } \\
\text { the MAIC } \\
\text { models }\end{array}$ \\
\hline Age & $\checkmark$ & & $\checkmark$ & $\checkmark$ & $\checkmark$ \\
\hline Sex & & & $\checkmark$ & $\checkmark$ & $\checkmark$ \\
\hline ECOG status & $\checkmark$ & & $\checkmark$ & $\checkmark$ & $\checkmark$ \\
\hline $\begin{array}{l}\text { Comorbidities } \\
\text { (renal, liver, or } \\
\text { frailty index) }\end{array}$ & $\checkmark$ & & & $\checkmark$ & $\checkmark$ \\
\hline $\begin{array}{l}\text { Cytogenetic } \\
\text { factors }\end{array}$ & $\checkmark$ & $\checkmark$ & $\checkmark$ & $\checkmark$ & $\checkmark$ \\
\hline R-ISS stage & $\checkmark$ & $\checkmark$ & $\checkmark$ & $\checkmark$ & $\checkmark$ \\
\hline $\begin{array}{l}\text { Extramedullary } \\
\text { disease }\end{array}$ & $\checkmark$ & & $\checkmark$ & & \\
\hline $\begin{array}{l}\text { Serum BCMA } \\
\text { levels }\end{array}$ & $\checkmark$ & & & & \\
\hline $\begin{array}{l}\text { Number of prior } \\
\text { lines of therapy }\end{array}$ & $\checkmark$ & $\checkmark$ & $\checkmark$ & $\checkmark$ & $\checkmark$ \\
\hline Refractory status & $\checkmark$ & $\checkmark$ & & $\checkmark$ & $\checkmark$ \\
\hline $\begin{array}{l}\text { Lytic bone lesions } \\
\text { at baseline }\end{array}$ & & & $\checkmark$ & & \\
\hline
\end{tabular}

BCMA, B-cell maturation antigen; ECOG, Eastern Cooperative Oncology Group; R-ISS, revised international staging system; MAIC, matching-adjusted indirect comparison

through independent clinical expert opinion and prognostic factors identified by exploratory analyses. Specifically, DREAMM-2 data were analyzed using Cox proportional hazards models to investigate the prognostic ability of several factors on OS, PFS, DoR, and TTR. Similarly, logistic regression models were used for ORR (Table 2). The base case MAIC model adjusted for between-study population differences in the following factors: age, sex, Eastern Cooperative Oncology Group (ECOG) performance status, creatinine clearance, revised international staging system, high cytogenetic risk (defined by either $\mathrm{t}[4 ; 14], \mathrm{t}[14 ; 16], 17 \mathrm{p} 13 \mathrm{del}$, or $1 \mathrm{q} 21+$ ), number of prior lines of therapy, and refractory status to last line of therapy. Two sensitivity MAICs were also carried out by repeating the
MAIC after adjusting for a different parametrization of ECOG (Sensitivity Model 1) or by adjusting for differences in the proportion of patients who were refractory to bortezomib, carfilzomib, lenalidomide, pomalidomide, and daratumumab (Sensitivity Model 2).

\section{Study Populations Included in ITC}

The comparative efficacy and safety of belamaf versus sel + dex were estimated using the weighted DREAMM-2 and the STORM Part 2 patient populations. For both the efficacy and safety analyses, MAIC weights were derived from the intention-to-treat (ITT) population of DREAMM-2 who received belamaf $2.5 \mathrm{mg} / \mathrm{kg}$ $(n=97)$ and who had creatinine levels reported 
$(n=95)$. The reported data for the ITT $(n=122)$ and safety $(n=123)$ populations of STORM Part 2 were used for population weighting $[9,16]$.

Patients from the MAMMOTH study were observed to receive SoC regimens, including combinations of daratumumab with an immunomodulatory drug or PI, elotuzumab with an immunomodulatory drug, carfilzomib with an immunomodulatory drug or an alkylator, or chemotherapy [8]. An ITC analysis was previously conducted using data from a subset of the MAMMOTH study $(n=128)$ [17]. This subset was selected to include only patients comparable with the STORM Part 2 study population, i.e., patients refractory to a PI, an immunomodulatory drug, and an anti-CD38 $\mathrm{mAb}$ who received anti-MM treatment other than sel + dex and were comparable with the STORM Part 2 study population [17].

\section{Outcome Measures}

Efficacy outcomes included: ORR, time to response (TTR), DoR, PFS, and OS of belamaf versus sel + dex or SoC. Tumor assessmentbased efficacy endpoints, such as ORR, TTR, DoR, PFS, and OS, were determined according to the IMWG criteria by an independent review committee in the DREAMM-2 trial.

Safety outcomes included any-grade treatment-emergent adverse events (TEAEs) in $\geq 5 \%$ of patients or Grade $3-4$ AEs in $\geq 5 \%$ of patients in either study. The proportions of patients who experienced certain TEAEs were compared between the DREAMM-2 and STORM Part 2 studies. These TEAEs included: thrombocytopenia, anemia, neutropenia, lymphopenia, leukopenia, fatigue, nausea, hyponatremia, pneumonia, diarrhea, hypokalemia, hyperglycemia, sepsis, mental status changes, or decreased appetite.

\section{Statistical Analyses}

HRs of belamaf versus sel + dex were derived using Cox regression model for time-to-event outcomes. Odds ratios (OR) of belamaf versus sel + dex were derived using logit models for overall response and safety outcomes. Statistical significance was assumed at $p<0.05$. Robust estimates of variance were used.

Comparative efficacy estimates of belamaf versus SoC were derived using a Bucher ITC using the HR reported in Costa et al.[17] (in which population differences were addressed by covariate adjustment) and the HR of belamaf versus sel + dex estimated in the MAIC.

\section{RESULTS}

\section{Systematic Literature Review}

The SLR identified 40 publications that reported data from 22 studies with late-line RRMM (seven randomized controlled trials, eight single-arm studies, four observational studies, and three pooled analyses of randomized controlled trials; Fig. 1). However, only the STORM (NCT02336815) Part 2 study [16] matched the inclusion criteria of the DREAMM-2 study and had similar study design and definitions of outcomes (as per IMWG 2016 efficacy criteria) [19].

\section{Population Weighting}

MAIC weights were derived separately for the efficacy and safety analyses of belamaf versus sel + dex. However, no weights could be derived for the two patients from the ITT population not included in the DREAMM-2 safety population because of missing values on some of the matching factors. Therefore, the MAIC weights produced for both the efficacy and safety analyses were identical. The baseline characteristics of the patients enrolled in DREAMM-2 before and after applying the MAIC weights and the corresponding characteristics for the STORM Part 2 patient population are presented in Table 3. Population adjustment was successful as the baseline characteristics of the weighted DREAMM-2 cohort matched the reported characteristics of the STORM Part 2 cohort for all the factors included in the MAIC. Following the MAIC adjustment, the ESS size was 63.46 patients, which corresponded to $65 \%$ of the original ITT population size. 


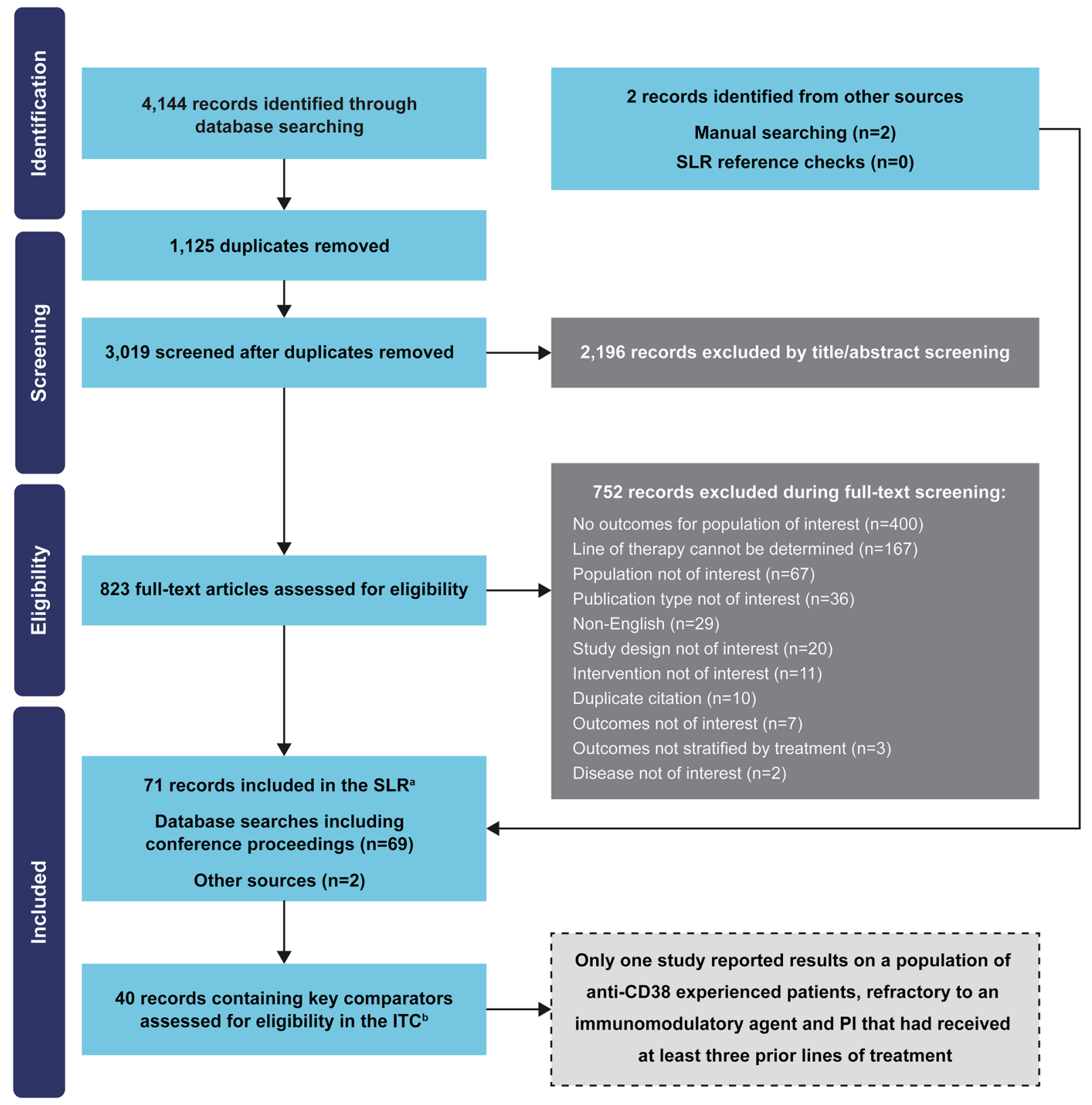

Fig. 1 PRISMA diagram. ${ }^{a}$ The scope of the SLR was wider than the inclusion criteria of the DREAMM-2 study in order to provide an overview of the treatment landscape. ${ }^{\mathrm{b}}$ Key comparators to belantamab mafodotin included the following treatments administered as mono- or combination therapies: bortezomib, carfilzomib, daratumumab, dexamethasone, elotuzumab, ixazomib, lenalidomide, pomalidomide, and selinexor. PI, proteasome inhibitor; RRMM, relapsed or refractory multiple myeloma; SLR, systematic literature review; ITC, indirect treatment comparisons 
Table 3 Baseline characteristics in the DREAMM-2 trial before and after MAIC adjustment plus baseline characteristics in the STORM Part 2 trial

\begin{tabular}{|c|c|c|c|c|}
\hline \multirow[t]{2}{*}{ Factor } & \multirow[t]{2}{*}{ Level } & \multicolumn{3}{|l|}{ Population } \\
\hline & & $\begin{array}{l}\text { DREAMM-2 } \\
\text { unadjusted data }\end{array}$ & $\begin{array}{l}\text { DREAMM-2 after } \\
\text { MAIC weighting }\end{array}$ & $\begin{array}{l}\text { sel }+ \text { dex observed in } \\
\text { STORM Part } 2\end{array}$ \\
\hline \multirow[t]{4}{*}{ Age, years } & Median & 65 & 64.4 & 65 \\
\hline & $51-64$ & $37.1 \%$ & $39.8 \%$ & $42.6 \%$ \\
\hline & $65-74$ & $40.2 \%$ & $36.1 \%$ & $36.1 \%$ \\
\hline & $\geq 75$ & $13.4 \%$ & $14.8 \%$ & $14.8 \%$ \\
\hline Sex & Male & $52.6 \%$ & $58.2 \%$ & $58.2 \%$ \\
\hline Race & White & $80.0 \%^{\mathrm{a}}$ & $76.7 \%$ & $69.9 \%$ \\
\hline \multirow[t]{3}{*}{ ECOG status } & 1 & $50.0 \%^{a}$ & $53.4 \%$ & $58.2 \%$ \\
\hline & 2 & $16.7 \%^{\mathrm{a}}$ & $13.8 \%$ & $9.0 \%$ \\
\hline & 1 or 2 & $66.7 \%^{a}$ & $67.2 \%$ & $67.2 \%$ \\
\hline \multirow[t]{3}{*}{ R-ISS stage } & II & $60.8 \%$ & $63.9 \%$ & $63.9 \%$ \\
\hline & III & $24.7 \%$ & $18.9 \%$ & $18.9 \%$ \\
\hline & II or III & $85.6 \%$ & $82.8 \%$ & $82.8 \%$ \\
\hline Cytogenetic risk & High-risk $^{\mathrm{b}}$ & $42.3 \%$ & $53.3 \%$ & $53.3 \%$ \\
\hline $\begin{array}{l}\text { Extramedullary } \\
\text { plasmacytomas }\end{array}$ & $\geq 1$ & $22.7 \%$ & $21.5 \%$ & Not reported \\
\hline Lytic bone lesion & Yes & $71.1 \%$ & $67.0 \%$ & Not reported \\
\hline Creatinine clearance & $\geq 60 \mathrm{ml} / \mathrm{min}$ & $72.0 \%^{a}$ & $66.4 \%$ & $66.4 \%$ \\
\hline \multirow{2}{*}{$\begin{array}{l}\text { Number of prior lines } \\
\text { of therapy }\end{array}$} & $\geq 5$ & $83.5 \%$ & $87.8 \%$ & $87.8 \%$ \\
\hline & $\geq 9$ & $17.5 \%$ & $29.3 \%$ & $29.3 \%$ \\
\hline Prior ASCT & Yes & $75.3 \%$ & $75.9 \%$ & $82.9 \%$ \\
\hline \multirow[t]{5}{*}{ Refractory status } & $\begin{array}{l}\text { To CFZ-POM- } \\
\text { DARA }\end{array}$ & $59.8 \%$ & $63.4 \%$ & $95.9 \%$ \\
\hline & $\begin{array}{l}\text { To BTZ-CFZ- } \\
\text { POM-DARA }\end{array}$ & $47.4 \%$ & $51.7 \%$ & $77.0 \%$ \\
\hline & $\begin{array}{l}\text { To LD-CFZ-POM- } \\
\text { DARA }\end{array}$ & $54.6 \%$ & $55.8 \%$ & $82.8 \%$ \\
\hline & $\begin{array}{l}\text { To BTZ-CFZ-LD- } \\
\text { POM-DARA }\end{array}$ & $42.3 \%$ & $44.1 \%$ & $68.0 \%$ \\
\hline & $\begin{array}{l}\text { To last line of } \\
\text { therapy }\end{array}$ & $95.7 \%^{\mathrm{a}}$ & $100.0 \%$ & $100.0 \%$ \\
\hline \multirow[t]{2}{*}{ Myeloma subtype } & $\operatorname{IgG}$ & $67.0 \%$ & $67.1 \%$ & $63.4 \%$ \\
\hline & Other heavy chain Ig & $24.7 \%$ & $24.2 \%$ & $14.6 \%$ \\
\hline
\end{tabular}


Table 3 continued

\begin{tabular}{|c|c|c|c|c|}
\hline \multirow[t]{2}{*}{ Factor } & \multirow[t]{2}{*}{ Level } & \multicolumn{3}{|l|}{ Population } \\
\hline & & $\begin{array}{l}\text { DREAMM-2 } \\
\text { unadjusted data }\end{array}$ & $\begin{array}{l}\text { DREAMM-2 after } \\
\text { MAIC weighting }\end{array}$ & $\begin{array}{l}\text { sel }+ \text { dex observed in } \\
\text { STORM Part } 2\end{array}$ \\
\hline $\begin{array}{l}\text { Bone marrow percent } \\
\text { plasma cells }\end{array}$ & $\geq 50 \%$ & $27.5 \%^{\mathrm{a}}$ & $26.0 \%$ & $22.8 \%$ \\
\hline
\end{tabular}

Bold font indicates characteristics included in the base case population-matching model

ASCT, autologous stem cell transplant; BTZ, bortezomib; CFZ, carfilzomib; DARA, daratumumab; ECOG, Eastern Cooperative Oncology Group; DoR, duration of response; Ig, immunoglobulin; LD, lenalidomide; MAIC, matchingadjusted indirect comparison; POM, pomalidomide; R-ISS, revised international staging system; sel + dex, selinexor plus dexamethasone; TTR, time to response

${ }^{a}$ Of non-missing observations

b The definition used to identify patients with high-risk cytogenetics was similar in both studies, i.e., patients with $t(4 ; 14)$, $\mathrm{t}(14 ; 16), 17 \mathrm{p} 13 \mathrm{del}$ or $1 \mathrm{q} 21+$

Table 4 MAIC of efficacy outcomes for belamaf versus sel + dex

\begin{tabular}{lll}
\hline Outcome $^{\mathrm{a}}$ & Naive estimates $(\mathbf{9 5 \%} \mathbf{C I})[\boldsymbol{p}$ value $]$ & Base case estimates $(\mathbf{9 5 \%} \mathbf{C I})[\boldsymbol{p}$ value $]$ \\
\hline ORR $^{\mathrm{b}}$ & OR: $1.32(0.73,2.38)[0.355]$ & OR: $1.00(0.52,1.91)[0.996]$ \\
DoR & HR: $\mathbf{0 . 4 1}(\mathbf{0 . 2 1}, \mathbf{0 . 8 3})[\mathbf{0 . 0 1 3}]$ & NA \\
TTR $^{\mathrm{c}}$ & HR: $0.65(0.39,1.10)[0.110]$ & HR: $0.71(0.43,1.15)[0.165]$ \\
PFS $^{\mathrm{c}, \mathrm{d}}$ & HR: $1.15(0.80,1.66)[0.438]$ & HR: $1.29(0.87,1.92)[0.199]$ \\
OS $^{\mathrm{c}}$ & HR: $\mathbf{0 . 6 0}(\mathbf{0 . 4 1}, \mathbf{0 . 8 8})[\mathbf{0 . 0 1 0}]$ & HR: $\mathbf{0 . 5 3}(\mathbf{0 . 3 4 , \mathbf { 0 . 8 3 }})[\mathbf{0 . 0 0 5}]$
\end{tabular}

Bold font indicates outcomes for which belamaf was significantly $(p<0.05)$ more efficacious than sel + dex Belamaf, belantamab mafodotin; CI, confidence interval; DoR, duration of response; HR, hazard ratio; MAIC, matchingadjusted indirect comparison; NA, not applicable; OR, odds ratio; ORR, overall response rate; OS, overall survival; PFS, progression-free survival; sel + dex, selinexor plus dexamethasone; TTR, time to response

${ }^{\mathrm{a}} \mathrm{HR}<1$ (except for TTR, HR $>1$ ) and OR $>1$ favor belamaf

b ORR was defined as achieving partial response or above

c $\mathrm{HR}$ should be interpreted with caution due to the crossing of the curves

d Suspicion of assessment-time bias

\section{Efficacy}

In the naive comparison, belamaf had a superior OS to sel $+\operatorname{dex}$ (HR 0.60; 95\% confidence interval [CI] 0.41, 0.88; $p=0.010$; Table 4). Following population weighting, the OS curve of the re-weighted belamaf cohort was shifted upwards (Fig. 2A), and the HR of belamaf versus sel + dex for OS improved to 0.53 (95\% CI 0.34, 0.83; $p=0.005$; Table 4).

Both before and after the population adjustment, patients treated with belamaf were found to achieve significantly longer DoR compared with sel + dex (Fig. $2 \mathrm{~B}$ and Table 4 ). In the naive comparison, belamaf had a longer DoR compared with sel + dex (HR 0.41; 95\% CI 0.21, $0.83 ; p=0.013$; Table 4). As DoR is measured from time of response rather than time from baseline, and DoR is interpretation based only on patients who respond to treatment rather than the full trial population, a MAIC conducted with weights that match full populations at baseline may be inappropriate. Acknowledging this limitation, an exploratory 

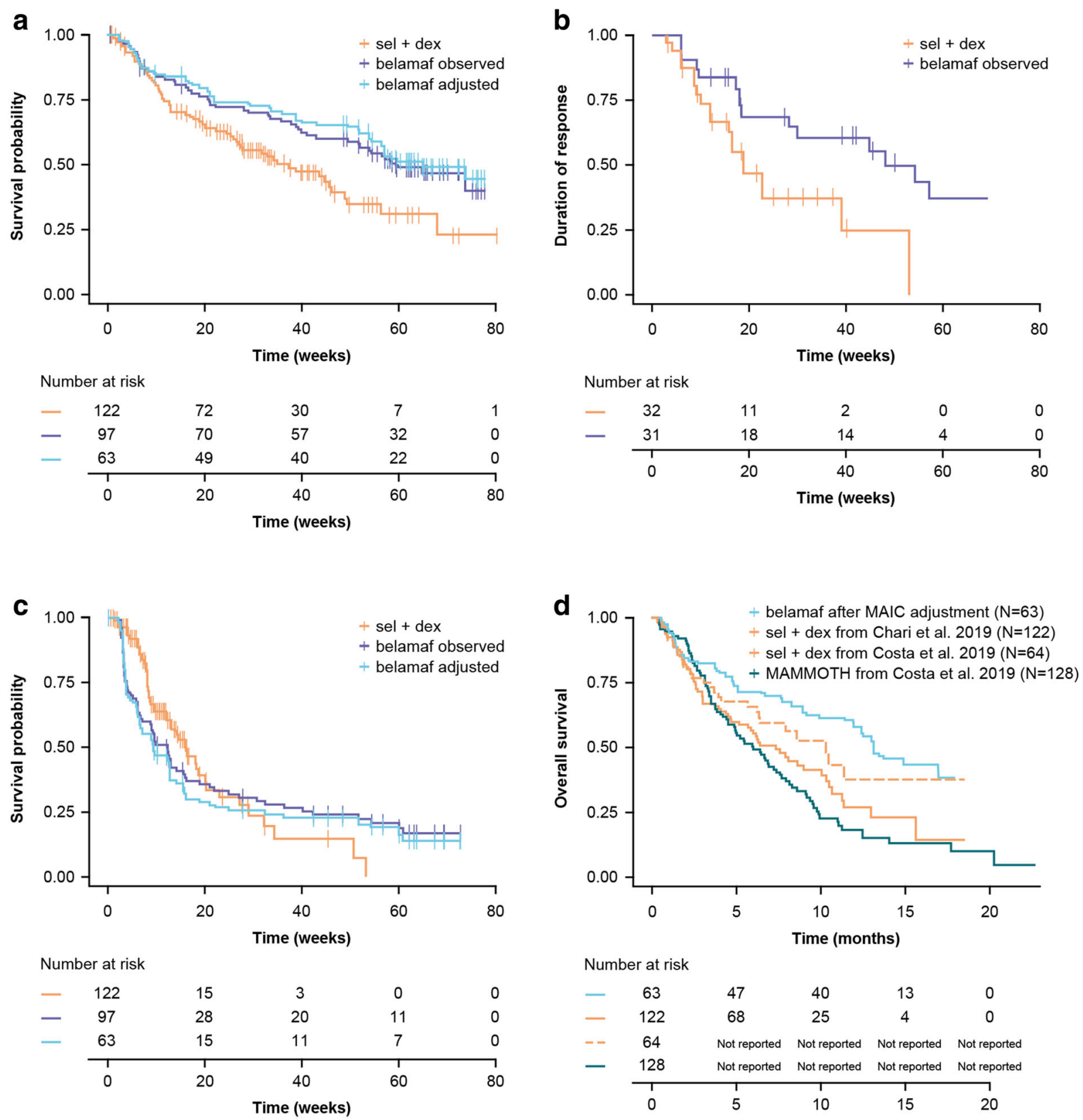

Fig. 2 OS (A), DoR (B) and PFS (C) Kaplan-Maier plots for belamaf $2.5 \mathrm{mg} / \mathrm{kg}$ (DREAMM-2) observed and MAIC adjusted versus sel + dex (STORM Part 2). (D) OS versus SoC from the MAMMOTH study (overlay of the estimates from different sources). Belamaf,

MAIC analysis was conducted and provided similar conclusions (HR 0.32; 95\% CI 0.13, 0.75; $p=0.009$; Supplementary Table 1).

The difference in PFS (Fig. 2C) and TTR between treatments was not statistically

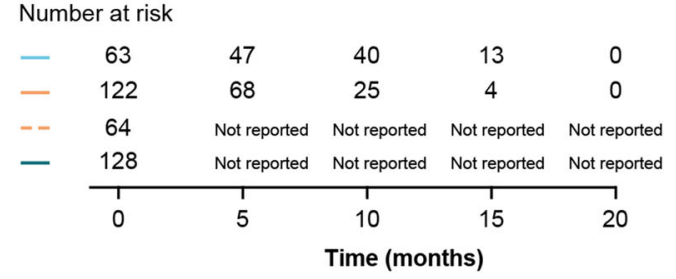

belantamab mafodotin; DoR, duration of response; MAIC, matching-adjusted indirect comparison; OS, overall survival; PFS, progression-free survival; sel + dex, selinexor plus dexamethasone

significant although numerically favorable HRs for sel + dex were observed. The HR for PFS was 1.29 (95\% CI 0.87, 1.92; $p=0.199)$ and for TTR was $0.71(95 \%$ CI $0.43,1.15$; $p=0.165)$. 


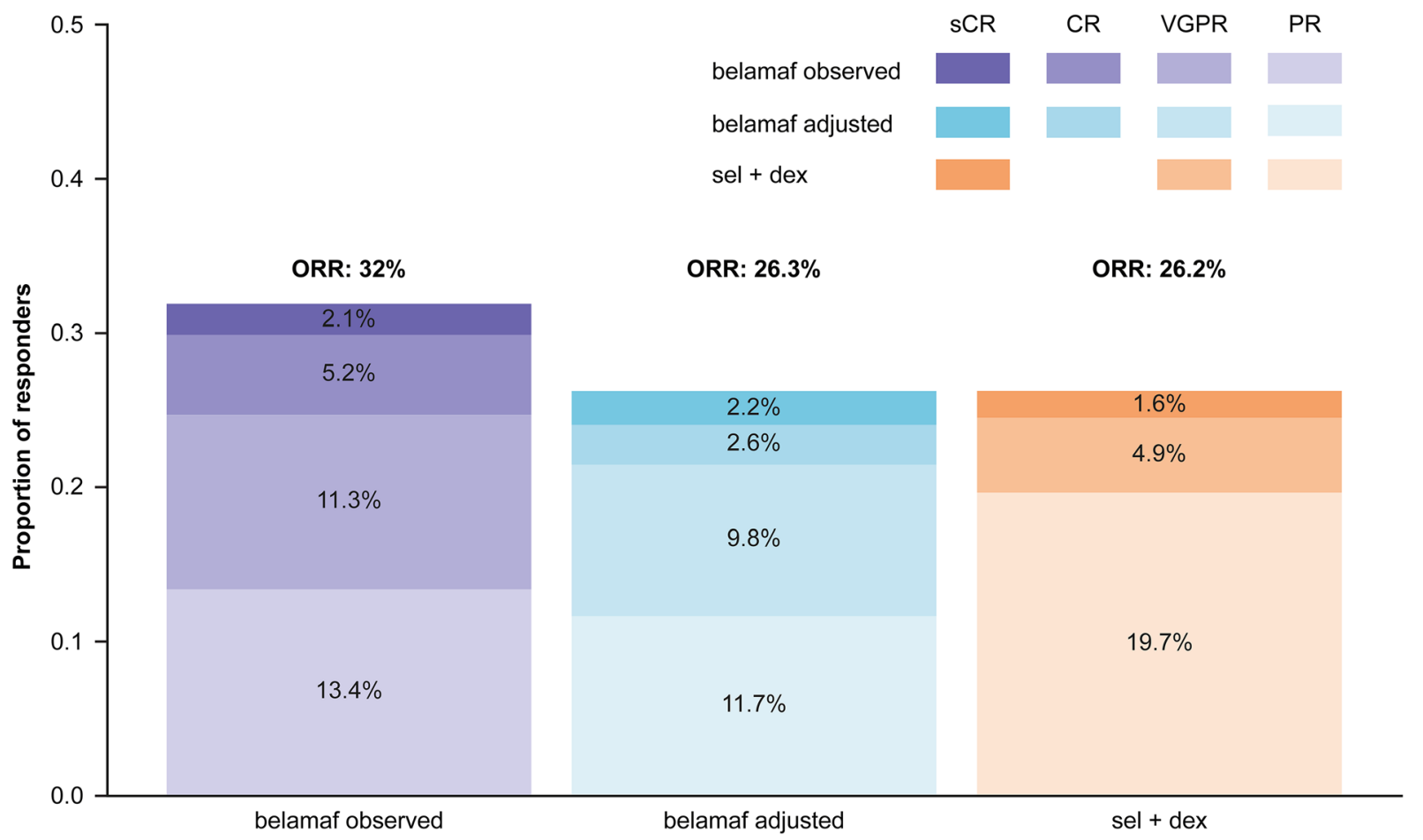

Fig. 3 Breakdown of patients per response type in the belamaf cohort before and after base case population adjustment from DREAMM-2 and in the observed sel + dex cohort from STORM Part 2. Belamaf, belantamab

Belamaf had a superior OS to SoC in MAMMOTH (Fig. 2D) in both Bucher analyses (i.e., with and without population matching in the comparison of belamaf versus sel + dex). The Bucher HR of belamaf versus sel + dex (using the MAIC adjusted HR versus sel + dex and covariate-adjusted HR of sel + dex versus MAMMOTH) was 0.29 (95\% CI 0.16, 0.54; $p<0.001)$ favoring belamaf. This was improved from 0.33 (95\% CI $0.18,0.54 ; p<0.001)$ in the Bucher analysis without population weighting in the comparison of belamaf versus sel + dex.

ORR values were not significantly different between the two treatments, with equivalent response rates found between belamaf and sel + dex (Fig. 3; Table 4). The adjusted OR was 1.00 (95\% CI 0.52, 1.91; $p=0.996$ ). Overall, $56 \%$ of responders had $\geq$ very good partial response with belamaf compared with $25 \%$ responders who were treated with sel + dex; $p=0.065$. mafodotin; $\mathrm{CR}$, complete response; $\mathrm{ORR}$, overall response rate; OS, overall survival; $\mathrm{PR}$, partial response; $\mathrm{sCR}$, stringent complete response; sel $+\mathrm{dex}$, selinexor plus dexamethasone; VGPR, very good partial response

Results across sensitivity analyses were consistent with the base case (Supplementary Table 1).

\section{Safety}

Compared with sel + dex, belamaf was found to have a significantly $(p<0.05)$ lower risk for most hematologic TEAEs, including any-grade and Grade 3-4 thrombocytopenia, anemia, and neutropenia as well as any-grade leukopenia (Fig. 4A). Belamaf and sel + dex were found to be equivalent in terms of risk for any-grade or Grade 3-4 lymphopenia.

ORs favored belamaf over sel + dex for most non-hematologic TEAEs (Fig. 4B). This difference was statistically significant $(p<0.05)$ for most TEAEs of any grade, with the exception of hyperglycemia and sepsis, which had a numerically lower (though not statistically significant) risk with belamaf. Hypercalcemia of any grade 
a

\section{Any-grade TEAEs}

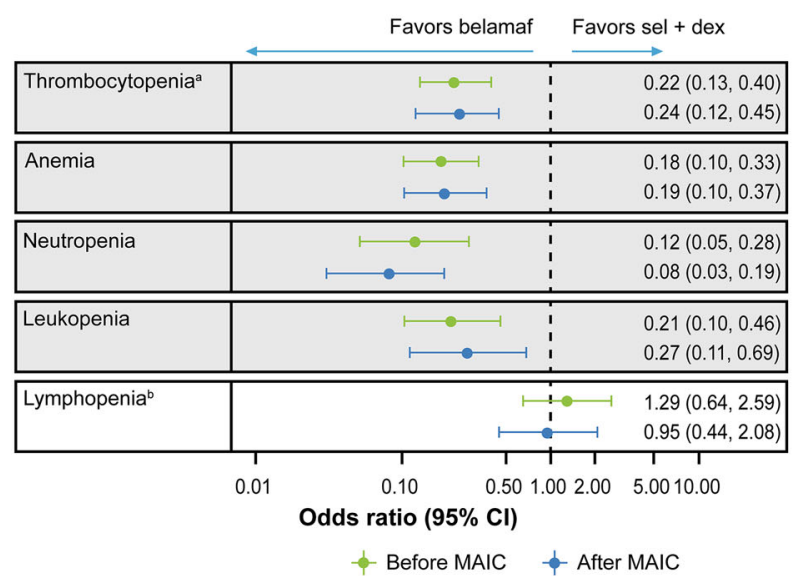

b

\begin{tabular}{|c|c|c|c|}
\hline \multicolumn{4}{|c|}{ Any-grade TEAEs } \\
\hline & Favors belamaf & \multicolumn{2}{|l|}{ Favors sel + dex } \\
\hline Fatigue $^{\circ}$ & $\longmapsto$ & $i$ & $\begin{array}{l}0.09(0.05,0.17) \\
0.07(0.03,0.15) \\
\end{array}$ \\
\hline Nausea & $\longmapsto$ & $i$ & $\begin{array}{l}0.13(0.07,0.25) \\
0.11(0.05,0.21) \\
\end{array}$ \\
\hline Hyponatremia & $\longmapsto$ & $i$ & $\begin{array}{l}0.12(0.05,0.29) \\
0.08(0.03,0.22) \\
\end{array}$ \\
\hline Pneumonia & $\longmapsto \longmapsto$ & & \begin{tabular}{|l|l|}
$0.57(0.25,1.28)$ \\
$0.37(0.14,0.97)$ \\
\end{tabular} \\
\hline Diarrhea & $\longmapsto$ & i & $\begin{array}{l}0.17(0.09,0.35) \\
0.15(0.07,0.34) \\
\end{array}$ \\
\hline Hypokalemia & 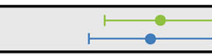 & & $\begin{array}{l}0.33(0.13,0.85) \\
0.28(0.10,0.82) \\
\end{array}$ \\
\hline Hyperglycemia & $\longmapsto$ & $\begin{array}{ll}3 \\
\vdots 1 \\
1-1\end{array}$ & $\begin{array}{l}0.47(0.16,1.38) \\
0.44(0.15,1.32) \\
\end{array}$ \\
\hline Sepsis & $\longmapsto$ & $: 1$ & $\begin{array}{l}.51(0.17,1.52) \\
0.38(0.11,1.35) \\
\end{array}$ \\
\hline $\begin{array}{l}\text { Mental status } \\
\text { changes }\end{array}$ & $\because \quad \longrightarrow$ & $\vdots$ & $\begin{array}{l}0.10(0.02,0.46) \\
0.12(0.03,0.58) \\
\end{array}$ \\
\hline $\begin{array}{l}\text { Decreased } \\
\text { appetite }\end{array}$ & $\longmapsto$ & $\vdots$ & $\begin{array}{l}0.11(0.06,0.23) \\
0.08(0.04,0.18) \\
\end{array}$ \\
\hline Hypercalcemia & & $\vdots \longmapsto$ & \begin{tabular}{|l|l|}
$5.14(1.63,16.26)$ \\
$4.57(1.39,15.07)$ \\
\end{tabular} \\
\hline & 0.10 & 10.00 & \\
\hline & Odds ratio & $(95 \% \mathrm{Cl})$ & \\
\hline & + Befor & $\phi$ After & \\
\hline
\end{tabular}

Fig. 4 Summary of the comparative safety of belamaf versus sel + dex before and after MAIC adjustment using all MAIC models. A Hematologic and B non-hematologic

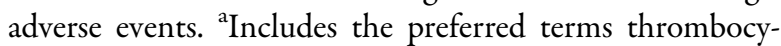
topenia, platelet count decreased; ${ }^{\text {includes the preferred }}$ terms lymphopenia, lymphocyte count decreased; 'includes the preferred terms fatigue and asthenia. OR $<1$ favors

was the only TEAE that was significantly more frequent with belamaf than with sel + dex. Patients treated with belamaf were also at significantly lower risk of developing Grade 3-4

\section{Grade 3-4 TEAEs}

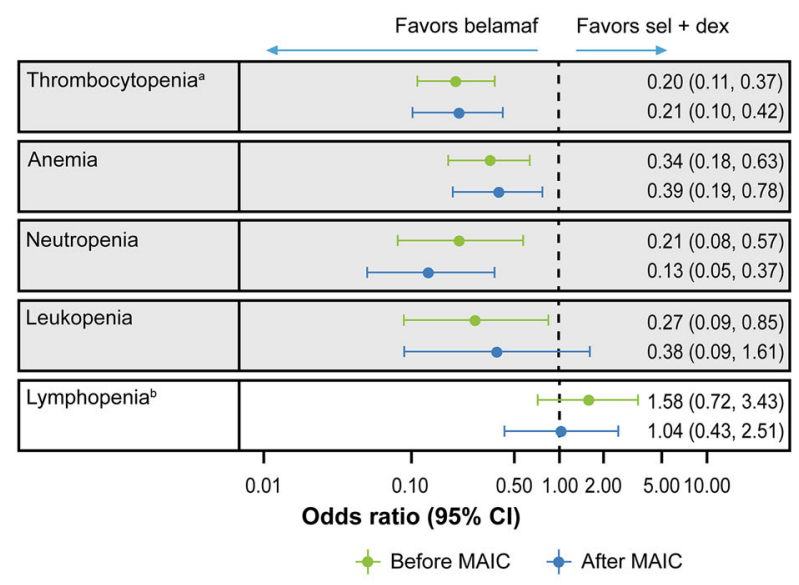

Grade 3-4 TEAEs

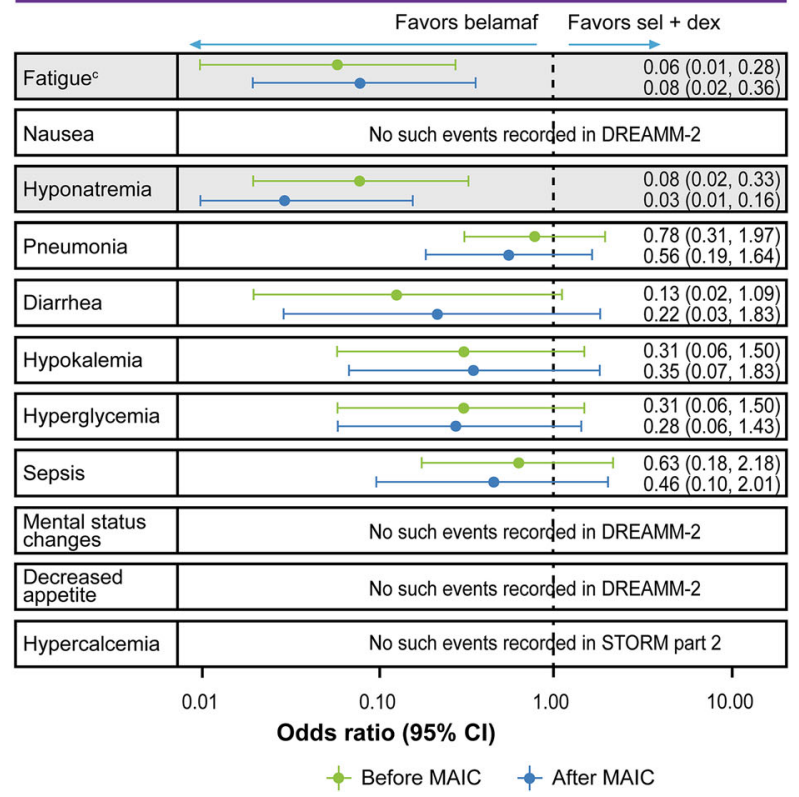

belamaf; OR $<0.5$, risk $50 \%$ lower with belamaf. TEAEs highlighted in gray boxes were significantly different. Belamaf, belantamab mafodotin; CI, confidence interval; MAIC, matching adjusted indirect comparison; sel + dex, selinexor plus dexamethasone; TEAE, treatment-related adverse events

fatigue, asthenia, or hyponatremia. Risk of Grade 3-4 pneumonias, diarrhea, hypokalemia, hyperglycemia, or sepsis was numerically (but not statistically) lower with belamaf. The 
sensitivity analysis results were similar to and supported the base case results (Supplementary Table 2).

Keratopathy (microcyst-like epithelial changes [MECs] defined as corneal epithelium changes identified on eye examination, with or without symptoms) was the most frequent TEAE in DREAMM-2 (any-grade keratopathy was observed in $72 \%$ of patients and Grade 3-4 in $46 \%$ of patients who received belamaf $2.5 \mathrm{mg} / \mathrm{kg}$; full details of keratopathy are described elsewhere[24]) but no such event has been reported in the STORM Part 2 study; therefore, an OR could not be derived. Similarly, no hypophosphatemia events were reported in the STORM Part 2 study, and therefore no comparative safety estimate could be derived.

\section{DISCUSSION}

The MAIC of belamaf (DREAMM-2) with sel + dex (STORM Part 2) was conducted following a SLR and searching of all relevant evidence. At the time of this research, STORM Part 2 was systematically identified as the only feasible comparator to the DREAMM-2 cohort. However, with the continuous development of new experimental therapies, more treatments may become available in the future requiring additional comparisons. The results of the MAIC analysis suggested that belamaf has a more favorable safety profile for most TEAEs, and patients treated with belamaf experienced a longer OS and DoR than those treated with sel + dex. It has been demonstrated that patients with RRMM typically experience shorter DoR with each subsequent therapy [6]. Therefore, sustaining longer responses with belamaf compared with sel + dex and SoC is particularly encouraging in patients who received $\geq 3$ prior therapies and whose MM was triple-class refractory to an immunomodulatory agent, a PI, and an anti-CD38 mAb. Response rates were found to be equivalent in terms of ORR between belamaf and sel + dex. TTR had a numerically worse efficacy profile with belamaf compared with sel + dex. However, the difference was not statistically significant. The steeper decline of the belamaf PFS curve around 4 weeks compared with sel + dex, combined with the similar response rates observed between belamaf and sel + dex, may suggest a faster progression among non-responding patients in the DREAMM-2 versus those in the STORM Part 2 study. However, this could also be attributed to differences in the time schedule of assessment; by trial design, the initial assessment for progressive disease (PD) happened earlier in the DREAMM-2 than the STORM, and therefore PD events were captured earlier in the DREAMM-2 compared with the STORM study.

In a single-arm study, OS, which measures death from any cause, can potentially be driven by other factors including subsequent treatments. The median PFS in the DREAMM-2 trial was 2.8 months $(95 \%$ CI $1.6,3.6)$ and median OS was 13.7 months (95\% CI 9.9, not reached [NR]) at the time of the January 2020 data cutoff $[9,11]$. In the $2.5 \mathrm{mg} / \mathrm{kg}$ cohort of the DREAMM-2 study, median PFS for the $35 \%$ of patients who had $\mathrm{PD} /$ not established (NE) response was 0.8 months $(95 \% \mathrm{CI} 0.7,0.8)$, and for the $31 \%$ of patients who achieved SD, median PFS was 2.9 months (95\% CI 2.1, 3.0). As displayed in Supplementary Fig. 1, this can be contrasted with median PFS NR (95\% CI 7.1, $\mathrm{NR}$ ) in $15 \%$ of patients who had $\mathrm{a} \geq$ minimal response (MR)/partial response (PR). Overall, 38 (39\%) patients received subsequent anticancer therapy (of these, only 2 received sel). The difference in OS outcomes by responder group is shown in Supplementary Fig. 2. Median OS was 8.7 months $(95 \%$ CI 1.9, 13.1.9) in patients who had PD/NE and 7.7 months (95\% CI 4.7, 13.4) in those who achieved SD. The median OS among patients with $\geq$ MR/PR was NR $(95 \%$ CI $\mathrm{NR}, \mathrm{NR})$. It is possible that some aspects of the observed survival benefit were driven by postprogression treatments. However, given the proportion of patients receiving subsequent anticancer treatments in the belamaf cohort and the magnitude of differences in outcomes between non-responders and responders, the difference seen in OS is likely to be driven by patients responding to belamaf treatment.

In general, belamaf had a more favorable safety profile than sel + dex for most evaluable hematologic and non-hematologic AEs, with the exception of hypercalcemia. These results 
were consistent across all models. As hypercalcemia is commonly reported in patients with $\mathrm{MM}$, the difference in incidence between belamaf and sel + dex may be related to disease progression rather than treatment $[19,25]$. In addition, dexamethasone used in the sel + dex combination could have had a calcium reduction effect via decreased intestinal calcium absorption. Similarly, dexamethasone may have contributed to the higher hyperglycemia rate in sel + dex. It should be noted that keratopathy was the most frequent treatment-associated $\mathrm{AE}$ in DREAMM-2, with $1 \%$ of patients in the $2.5 \mathrm{mg} / \mathrm{kg}$ cohort discontinuing treatment as a result [11]. Keratopathy was managed with dose modification $(47 \%$ of patients had dose delays and $25 \%$ had dose reductions in the $2.5 \mathrm{mg} / \mathrm{kg}$ cohort). Ocular events are known side effects of MMAF-containing ADCs [26]. No keratopathy and hypophosphatasemia events were reported in the STORM Part 2 study so no statistical comparison could be made between the two treatments [9, 16, 24].

In the absence of head-to-head randomized controlled trials, population-adjusted ITCs can be valuable tools to compare efficacy and safety of treatments from separate studies to inform clinical practice and value analyses. However, it is crucial that the included clinical trials have similar patient populations, design, and definitions. In this study, the weighting process for all of these aspects was successful. The ESS achieved was considered satisfactory (65\% of original sample size), and there were no extreme MAIC weights, which ensured that the results were not affected disproportionately by only a few patients. This notion was further supported by the similar trend of time to events both before and after the population adjustment.

The current MAIC analyses are subject to potential limitations relating to the comparability of studies. Although both studies had similar trial designs, and population characteristics were weighted successfully, differences in the frequency of assessment for PD between the two studies may have introduced bias in these unanchored ITC. In the STORM Part 2 study, the response and PD assessments were performed on a 4-weekly schedule, while in the DREAMM-2 study, patients were monitored on a 3-weekly schedule. As PFS and TTR were recorded at different scheduled monitoring visits in each study, unanchored comparisons of PFS and TTR may be subject to assessment time bias [27].

Additionally, differences in unobserved patient baseline characteristics can confound comparisons despite matching populations on observed characteristics. Limited data were available for the STORM Part 2 study population on frailty of patients at baseline. Furthermore, certain prognostic factors were not reported in STORM Part 2 (extramedullary disease at baseline, BCMA levels, presence of lytic bone lesions at baseline) and could not be included in the MAIC models (Supplementary Table 1). The two studies could not be balanced for time since diagnosis or mutation-specific factors because of missing data. Different levels of prognostic factors with similar prognosis of outcomes were combined to increase ESS after MAIC weighting, as matching distributions at a more granular level required a larger reduction in the effective sample. Additionally, as the proportion of patients that were refractory to a variety of combinations of active drugs was higher in STORM Part 2 compared with the DREAMM-2 study, the results should be interpreted with caution. However, a sensitivity analysis in which the proportion of patients with pentarefractory disease at baseline were matched across both studies provided similar results (Supplementary Table 1). Furthermore, there was a single trial to inform the comparison between belamaf and sel + dex. If more trials were available for MAIC, HRs for each comparator could have been pooled. Despite these limitations, the MAIC methodology was successfully applied to compare belamaf versus sel + dex and suggests a significant difference in OS, DoR, and most AEs in favor of belamaf.

The ITC of belamaf versus SoC suggests that belamaf significantly prolongs OS over SoC. This analysis relies on the assumption that the two HRs that were compared, i.e., the HR comparing sel + dex versus SoC in Costa et al. [17] and the HR comparing belamaf versus sel + dex after population weighting of the DREAMM-2 and STORM Part 2 populations, are independent from the population in which 
they have been measured and can, therefore, be compared. We could find no evidence suggesting that this assumption does not hold. In addition, the adjusted HR of sel + dex versus SoC from the MAMMOTH study could be confounded by the use of real-world studies in the comparison. A final consideration is that patients included in the MAMMOTH study may have been excluded from participation in clinical trials because of their fragile health status.

\section{CONCLUSION}

In conclusion, single-agent belamaf represents a new treatment option for multiply relapsed patients with RRMM. In these analyses, the ITC that used MAIC based on the 13-month followup of the DREAMM-2 study found belamaf to be significantly more efficacious than sel + dex in terms of OS and DoR. A significantly prolonged OS was also estimated for belamaf compared with SoC, as observed in the MAMMOTH study. The results also revealed a more favorable safety profile for belamaf than sel + dex, as demonstrated by significantly lower incidence of anygrade and Grade 3-4 hematologic AEs (with the exception of lymphopenia) and of most anygrade non-hematologic AEs including fatigue, nausea, hyponatremia, pneumonia, diarrhea, hypokalemia, mental status changes, and decreased appetite. Keratopathy (MECs) was the most common TEAE in DREAMM-2 but was not reported in STORM Part 2. Further comparisons of efficacy and safety can be carried out if suitable data become available.

\section{ACKNOWLEDGEMENTS}

Funding. We thank the participants of the study. This study was funded by GlaxoSmithKline (GSK; 207147). GSK contributed to the study design, implementation, data collection, interpretation, and analysis.

Medical Writing and Editorial Assistance. Medical writing support was provided by Joanna Nikitorowicz-Buniak, PhD, of Fishawack
Indicia Ltd., UK, and funded by GSK. GSK is also funding the Open Access Fee for this manuscript. Drug linker technology was licensed from Seagen Inc (Bothell, WA, USA), and the monoclonal antibody was produced with POTELLIGENT Technology licensed from BioWa (Princeton, NJ, USA). RP is supported by the National Institute for Health Research University College London Hospitals Biomedical Research Centre. AS is supported by the Veterans Affair merit award (BX004514).

Authorship. All named authors meet the International Committee of Medical Journal Editors (ICMJE) criteria for authorship for this article, take responsibility for the integrity of the work as a whole, and have given their approval for this version to be published.

Authorship Contributions. All authors made substantial contributions to the conception or design of the work; or the acquisition, analysis, or interpretation of data for the work; were responsible for drafting the work or revising it critically for important intellectual content; provided final approval of the version to be published.

Prior Presentation. Part of the data reported in this manuscript were presented as an abstract at the ASCO 2020 Congress and as posters at the European Haematology Association (EHA) 2020 Congress, annual meeting of the Society of Hematologic Oncology (SOHO) 2020, and the European Society for Medical Oncology (ESMO) virtual congress 2020.

Disclosures. Thibaud Prawitz, Rachel Hughes, and Venediktos Kapetanakis are employees of Evidera, which received research funding from GSK. Rakesh Popat has received consulting fees from AbbVie, Celgene, GSK, and Takeda; personal fees from GSK, Janssen, and Takeda; honoraria from Celgene, GSK, Janssen, and Takeda, and research funding from Takeda. Attaya Suvannasankha has received consulting fees from GSK, Janssen, and Karyopharm Therapeutics; research funding from BristolMyers Squibb, Celgene, GSK, and Janssen; and personal fees from GSK and Janssen. Grammati 
Sarri was an employee of Evidera at the time of study design and initiation. Feng Wang, Cosmina Hogea, Boris Gorsh, and Jenny Willson are employees of and have stocks and shares in GSK. Shannon Allen Ferrante is an employee of and has stocks and shares in GSK, and has shares in Merck.

Compliance with Ethics Guidelines. This article is based on previously conducted studies and does not contain any studies with human participants or animals performed by any of the authors.

Data Availability. GSK makes available anonymized individual participant data and associated documents from interventional clinical studies which evaluate medicines, upon approval of proposals submitted to http://www. clinicalstudydatarequest.com. To access data for other types of GSK sponsored research, for study documents without patient-level data and for clinical studies not listed, please submit an enquiry via the website.

Open Access. This article is licensed under a Creative Commons Attribution-NonCommercial 4.0 International License, which permits any non-commercial use, sharing, adaptation, distribution and reproduction in any medium or format, as long as you give appropriate credit to the original author(s) and the source, provide a link to the Creative Commons licence, and indicate if changes were made. The images or other third party material in this article are included in the article's Creative Commons licence, unless indicated otherwise in a credit line to the material. If material is not included in the article's Creative Commons licence and your intended use is not permitted by statutory regulation or exceeds the permitted use, you will need to obtain permission directly from the copyright holder. To view a copy of this licence, visit http://creativecommons.org/licenses/by$\mathrm{nc} / 4.0 /$.

\section{REFERENCES}

1. Kazandjian D. Multiple myeloma epidemiology and survival: a unique malignancy. Semin Oncol. 2016;43:676-81.

2. Robak P, Drozdz I, Szemraj J, Robak T. Drug resistance in multiple myeloma. Cancer Treat Rev. 2018;70:199-208.

3. Rajkumar SV, Kumar S. Multiple myeloma: diagnosis and treatment. Mayo Clin Proc. 2016;91: 101-19.

4. Podar K, Shah J, Chari A, Richardson PG, Jagannath S. Selinexor for the treatment of multiple myeloma. Expert Opin Pharmacother. 2020;21:399-408.

5. Sonneveld P, De Wit E, Moreau P. How have evolutions in strategies for the treatment of relapsed/ refractory multiple myeloma translated into improved outcomes for patients? Crit Rev Oncol Hematol. 2017;112:153-70.

6. Kumar SK, Therneau TM, Gertz MA, et al. Clinical course of patients with relapsed multiple myeloma. Mayo Clin Proc. 2004;79:867-74.

7. Yong K, Delforge M, Driessen C, et al. Multiple myeloma: patient outcomes in real-world practice. Br J Haematol. 2016;175:252-64.

8. Gandhi UH, Cornell RF, Lakshman A, et al. Outcomes of patients with multiple myeloma refractory to CD38-targeted monoclonal antibody therapy. Leukemia. 2019;33:2266-75.

9. Lonial S, Lee HC, Badros A, et al. Belantamab mafodotin for relapsed or refractory multiple myeloma (DREAMM-2): a two-arm, randomised, openlabel, phase 2 study. Lancet Oncol. 2020;21:207-21.

10. Tai YT, Anderson KC. Targeting B-cell maturation antigen in multiple myeloma. Immunotherapy. 2015;7:1187-99.

11. Lonial $\mathrm{S}$, Lee $\mathrm{H}$, Badros $\mathrm{A}$, et al. The American Society for Clinical Oncology (ASCO) Congress; May 29-31, 2020; Virtual Format: ASCO; 2020.

12. GlaxoSmithKline, Research Triangle Park, NC. BLENREP prescribing information. 2020. https:// gsksource.com/pharma/content/dam/ GlaxoSmithKline/US/en/Prescribing_Information/ Blenrep/pdf/BLENREP-PI-MG.PDF.

13. European Medicines Agency (EMA). BLENREP 100 mg powder [Summary of Product Characteristics]; 2020. https://www.ema.europa.eu/en/documents/ product-information/blenrep-epar-productinformation_en.pdf. 
14. Phillippo DM, AE A, Dias S, Palmer S, Abrams KR, Welton NJ. NICE DSU technical support document 18: methods for population-adjusted indirect comparisons in submissions to NICE. 2016. http://www. nicedsu.org.uk/wp-content/uploads/2017/05/ Population-adjustment-TSD-FINAL.pdf. Accessed 2 Apr 2020.

15. Hughes R, Sarri G, Kapetanakis V, et al. Clinical outcomes in late-line relapsed/refractory multiple myeloma: systematic literature review [unpublished manuscript]. Evidera, San Francisco, CA, USA 2021.

16. Chari A, Vogl DT, Gavriatopoulou M, et al. Oral selinexor-dexamethasone for triple-class refractory multiple myeloma. N Engl J Med. 2019;381:727-38.

17. Costa LJ, Hari P, Kumar SK, et al. Overall survival of triple class refractory, penta-exposed multiple myeloma (MM) patients treated with selinexor plus dexamethasone or conventional care: a combined analysis of the STORM and mammoth studies. Blood. 2019;134:3125.

18. Bucher HC, Guyatt GH, Griffith LE, Walter SD. The results of direct and indirect treatment comparisons in meta-analysis of randomized controlled trials. J Clin Epidemiol. 1997;50:683-91.

19. Kumar S, Paiva B, Anderson KC, et al. International Myeloma Working Group consensus criteria for response and minimal residual disease assessment in multiple myeloma. Lancet Oncol. 2016;17: e328-46.

20. Vogl DT, Dingli D, Cornell RF, et al. Selective inhibition of nuclear export with oral selinexor for treatment of relapsed or refractory multiple myeloma. J Clin Oncol. 2018;36:859-66.

21. Chari A, Vogl DT, Dimopoulos M, et al., editors. Results of the pivotal STORM study (Part 2): deep and durable responses with oral selinexor plus low dose dexamethasone in patients with penta- exposed and triple class refractory MM. 62nd American Society of Hematology Annual Meeting \& Exposition 2018; San Diego, CA, USA.

22. Jagannath S, Vogl DT, Dimopoulos M, et al., editors. Efficacy of oral selinexor plus low dose dexamethasone ( $\mathrm{Sd}$ ) in patients with penta-refractory myeloma: results of the pivotal STORM Part II Study. 6th Annual Meeting: Society of Hematologic Oncology; 2018; Houston, TX, USA.

23. Food and Drug Administration (FDA). Multi-discipline review of selinexor (proposed trade name: $\mathrm{XPOVIO}^{\circledR}$ ) application number 212306 Orig1s000; 2019. https://www.accessdata.fda.gov/drugsatfda docs/nda/2019/212306Orig1s000MultidisciplineR. pdf. Accessed Apr 2020.

24. Farooq AV, Degli Esposti S, Popat R, et al. Corneal epithelial findings in patients with multiple myeloma treated with antibody-drug conjugate belantamab mafodotin in the pivotal, randomized, DREAMM-2 study. Ophthalmol Ther. 2020;9(4): 889-911.

25. Gastanaga VM, Schwartzberg LS, Jain RK, et al. Prevalence of hypercalcemia among cancer patients in the United States. Cancer Med. 2016;5:2091-100.

26. Eaton JS, Miller PE, Mannis MJ, Murphy CJ. Ocular adverse events associated with antibody-drug conjugates in human clinical trials. J Ocul Pharmacol Ther. 2015;31:589-604.

27. Kapetanakis V, Prawitz T, Schlichting M, et al. Assessment-schedule matching in unanchored indirect treatment comparisons of progression-free survival in cancer studies. Pharmacoeconomics. 2019;37:1537-51.

28. Rajkumar SV. Updated diagnostic criteria and staging system for multiple myeloma. Am Soc Clin Oncol Educ Book. 2016;35:e418-23. 